Aus der I. Universitätsklinik für Chirurgie (Vorstand: Prof. Dr. A. Fritsch), Wien

\section{Eingeladener Kommentar: \\ Untersuchungen über den Immunstatus bei langzeitiberlebenden Empfängern von allogenen Dünndarmtransplantaten im Rattenmodell}

\section{F. Mühlbacher}

Dem Phänomen Toleranz begegnet man in der gesamten Biologie: Beginnend bei der Reaktionslosigkeit des Immunsystems gegenüber körpereigenen antigenen Strukturen (Selbsttoleranz), ïber die induzierte Toleranz gegenüber Alloantigenen, wie sie im Dünndarmtransplantationsmodell der Ratte (1) durch temporäre Immunsuppression erreicht wurde, bis zu jener pharmakologisch unterhaltenen Toleranz, wie wir sie der zur Routine gewordenen humanen Organtransplantation kennen. So verschieden wie die Erscheinungsformen dürften auch die der Toleranz zugrundeliegenden Mechanismen sein. Das Konzept der Toleranz gegenüber eigenen Antigenen wurde zuerst von Burnet 1959 (2) im Sinne der "Clone Deletion" erklärt, dafür gibt es eine Serie von modemen Experimenten, $\mathrm{da}$ diese Clone Deletion im Thymus erfolgt und zur Selbsttoleranz führt (3).

Die Induktion von Toleranz ist im Tiermodell an erwachsenen Individuen möglich. So z. B. durch spenderspezifische Transfusionen an der Ratte, durch vorübergehende Gabe von Cyclosporin (1) oder FK 506, durch Lebertransplantation ohne Immunsuppression in der Ratte (4), Maus (5) und vielen ähnlichen Experimenten. Es erhebt sich zwangsläufig die Frage, nach welchem Mechanismus diese Toleranz induziert wurde. Aus dem vorliegenden Experiment der Gruppe um Langrehr konnte klar nachgewiesen werden, daß die durch Cyclosporin induzierte Toleranz gegenüber dem allogenen Dünndarmtransplantat nicht nach der Clone-DeletionTheorie funktioniert: Die Lymphozyten der Empfängertiere sind sowohl durch donorspezifische Antigene als auch durch Drittantigen normal zur Proliferation stimulierbar, zusätzlich behalten diese Zellen ihre zytotoxische Aktivität wieder sowohl gegen spenderspezifische Antigene als auch gegen ein Drittantigen. Dieses Experiment hat also die Clone-Deletion-Theorie als Mechanismus für diese Toleranzinduktion widerlegt. Interessant erscheinen mir die so im Nebenzug durchgeführten morphologischen Untersuchungen des transplantierten Dünndarms: Der gesamte

Korrespondenzanschrift: Prof. Dr. F. Mühlbacher, I. Universitätsklinik für Chirurgie. Alser Straße 4, A-1090 Wien. lymphatische Apparat ist verschwunden und durch Narben ersetzt, es finden sich vereinzelt lymphozytäre Zellinfiltrate deren Abstammung in diesem Experiment nicht untersucht wurde. Wahrscheinlich liegt hier der Schlüsselpunkt der Frage. Wenn schon nicht die Abwehrlage des Wirtsorganismus modifiziert worden ist, so muß die Antigenität des Transplantats modifiziert worden sein. Grundlach et al. (6) konnten zeigen, daß es beim Toleranzphänomen sowohl im Dünndarm als auch bei Herztransplantaten zu einer Depletion von Passenger-Zellen und dendritischen Zellen kommt, so daß die Antigenpräsentation im Wirtsorganismus offensichtlich nicht mehr möglich ist. Es dürfte sich also um eine aktiv unterhaltene Form der Suppression handeln $(7,8)$, die möglicherweise durch lymphozytäre Elemente mit Suppressorfunktion aufrechterhalten wird. Experimente mit adoptivem Transfer von toleranten Tieren innerhalb syngener Individuen sprechen zusätzlich für diese Hypo-

Kongreßankündigungen

8. Berliner Gefäßchirurgisches Symposium

Termin und Ort: 11. bis 13. November 1993 - Berlin

Organisation und Auskuntt: Prof. Dr. W. Hepp, Gefäßchirurgie, Oskar-HeleneHeim, Clayallee 229, D-W-1000 Berlin 33, Tel. 06 / 030 / 81004 - 387.

Fax: $06 / 030 / 8814925$.

$10^{\text {th }}$ Congress - Intemational Society for Laser Surgery and Medicine $7^{\text {th }}$ International YAG Laser Symposium

Termin und Ort: 12. bis 17. November 1993 - Bangkok, Thailand.

Antragen und Anmeldungen: C.C.E. (Thailand), 248 Ladprao Road (47), Bangkapi, Bangkok 10 310, Tel. 00662 / 538 - 53 32, 481 - 88 09, Fax 00662 / $538-5332$.

DIVI 93 - 2 Kongre 3 der Deutschen Interdisziplinären

Vereinigung für Intensiv- und Notfallmedizin (DIV)

Termin und Ort: 24. bis 27. November 1993 - Hamburg, Congress Centrum. Kongreßsekretariat: DIVI '93, Congress Centrum Hamburg, Congress Organisation, Jungiusstraße 13, Postfach 302480 , D-W-2000 Hamburg 36. Tel. $06 / 040 / 3569-2245$

Symposium: Kolorektales Karzinom - Standards in Diagnose und therapie

Termin und Ort: 27. bis 29. Jänner 1994 - Erlangen.

Organisation und Sekretariat: Chirurgische Klinik und Poliklinik der Universität Erlangen-Nürnberg, Maximiliansplatz, D-W-8520 Erlangen, Tel. $06 / 09131 /$ 8535 58. Frau H. Scipio, Fax: 06/09131/85 4675. 